\title{
The ripple effect of a complication in lung transplantation: Evidence for increased long-term survival risk
}

\author{
Ernest G. Chan, MD, ${ }^{\mathrm{a}}$ Valentino Bianco III, DO, MPH, ${ }^{\mathrm{a}}$ Thomas Richards, $\mathrm{PhD},{ }^{\mathrm{a}}$ \\ J. W. Awori Hayanga, MD, MPH, ${ }^{a}$ Matthew Morrell, MD, ${ }^{b}$ Norihisa Shigemura, MD, PhD, ${ }^{a}$ \\ Maria Crespo, MD, ${ }^{\mathrm{b}}$ Joseph Pilewski, MD, ${ }^{\mathrm{b}}$ James Luketich, $\mathrm{MD},{ }^{\mathrm{a}}$ and Jonathan $\mathrm{D}^{\prime}$ Cunha, MD, PhD ${ }^{\mathrm{a}}$
}

\begin{abstract}
Objective: Lung transplantation is a life-saving procedure for patients who have end-stage lung disease. The frequency and severity of complications have not been fully characterized. We hypothesized that early in-hospital, postoperative complications decrease long-term survival.
\end{abstract}

Methods: We retrospectively identified in-hospital complications in lung transplant recipients, from the period January 2007 to October 2013. Complications were graded using the extended Accordion Severity Grading System (ASGS). Complications were categorized by event and organ system. Survival analysis was performed $(P<.05)$ using a time-dependent model.

Results: Among 748 eligible patients, 3381 independent in-hospital, postoperative complications occurred in $92.78 \%$ of patients. Median follow-up was 5.4 years. Complications associated with significant decrease in 5-year survival were: renal (hazard ratio [HR] 2.58, 95\% confidence interval [CI] 1.40-4.48); hepatic (HR 4.08, 95\% CI 2.86-5.82); cardiac (HR 1.95, 95\% CI 1.56-2.45). The maximum ASGS of $\geq 5(18.5 \%$ vs $73.8 \%)$, and the weighted ASGS sum $>10(2.5 \%$ vs $73.8 \%)$, were found to be significant predictors of long-term survival. Multivariate analysis identified a weighted ASGS sum of $>10$, and renal, cardiac, and vascular complications as predictors of decreased long-term survival.

Conclusions: Rigorous delineation of complications after lung transplantation showed that grade 5 ASGS in-hospital postoperative complications, and a weighted ASGS sum $>10$, were independent predictors of decreased long-term survival well beyond the initial perioperative period. These results may identify important targets for best practice guidelines and quality-of-care measures after lung transplantation. (J Thorac Cardiovasc Surg 2016;151:1171-80)

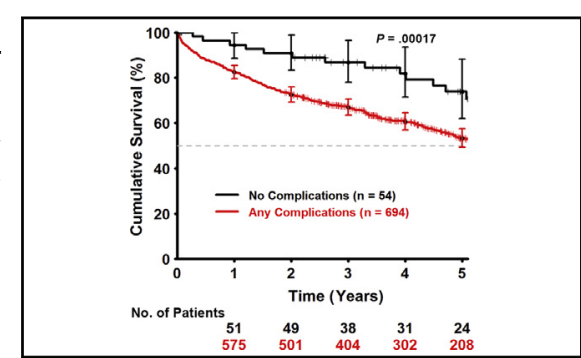

Time-dependent survival curves for patients who have any postoperative complications, versus none.

\section{Central Message}

Grade $\geq 5$ ASGS in-hospital postoperative complications, and a weighted ASGS sum of $>10$ were independent predictors of long-term survival.

\section{Perspective}

The overall 90-day postoperative course influences long-term survival. Both severity of the intervention needed to overcome a complication, and having many less-severe complications, have negative effects on long-term survival. These results support the need to establish best-practice guidelines outlining the best way to avoid complications, particularly during the initial period.

See Editorial Commentary page 1181.
Advancements in operative techniques and postoperative management have greatly evolved and served to improve survival outcomes after lung transplantation surgery during the past 3 decades. ${ }^{1,2}$ Despite these improvements, however, 5-year survival of patients undergoing lung transplantation remains in the range of $55 \%$. Some argue ${ }^{3}$ that

From the Departments of ${ }^{\mathrm{a}}$ Cardiothoracic Surgery and ${ }^{\mathrm{b}}$ Pulmonary Medicine, University of Pittsburgh Medical Center, Pittsburgh, Pa.

Read at the 41st Annual Meeting of The Western Thoracic Surgical Association, Whistler, British Columbia, Canada, June 24-27, 2015.

Received for publication June 22, 2015; revisions received Nov 6, 2015; accepted for publication Nov 25, 2015; available ahead of print Jan 20, 2016.

Address for reprints: Jonathan D'Cunha, MD, PhD, Department of Cardiothoracic Surgery, University of Pittsburgh Medical Center, UPMC Presbyterian Ste C-900 200, Lothrop St, Pittsburgh, PA 15213 (E-mail: dcunhaj@upmc.edu). $0022-5223 / \$ 36.00$

Copyright (c) 2016 by The American Association for Thoracic Surgery http://dx.doi.org/10.1016/j.jtcvs.2015.11.058 survival rates have plateaued in 2005 , owing to a change in candidate selection from the implementation of the lung allocation score. Nevertheless, our goal should be to identify methods in which we can improve medical care given to these patients to push the field forward and improve outcomes.

An evolving literature suggests that postoperative course is correlated with long-term survival. ${ }^{4,5}$ Highvolume centers performing lung resection surgery have decreased perioperative morbidity rates, compared with

Scanning this QR code will take you to the article title page.

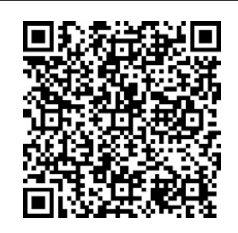




\section{Abbreviations and Acronyms \\ ASGS $=$ Accordion Severity Grading System \\ $\mathrm{CCI}=$ Charlson Comorbidity Index \\ $\mathrm{CI}=$ confidence interval \\ $\mathrm{HR}=$ hazard ratio \\ ICD-9 $=$ International Classification of Diseases, 9 th edition}

lower-volume centers, indicating potentially more-effective management of postoperative complications that affect long-term survival. Using the American College of Surgeons National Surgical Quality Improvement Program Database, Ghaferi and colleagues ${ }^{6,7}$ attributed a 2.5-foldhigher mortality rate in the "worst" hospitals to their much higher failure-to-rescue rates. They proposed standardization of the management of postoperative complications as an effective strategy in decreasing variability in mortality after major surgery, across institutions. ${ }^{6,7}$

In addition, center volume may have a strong association with improved survival, even beyond the initial perioperative period of 30 days in many fields, including thoracic surgery and transplantation. ${ }^{8-12}$ Recently, our group demonstrated a negative association between in-hospital complications and 5-year overall and cancer-specific survival in patients undergoing lung resection surgery for stage I non-small cell lung cancer using the Surveillance, Epidemiology, and End Results (SEER) database. ${ }^{5}$ Others ${ }^{10}$ have demonstrated equivalent incidences of individual postoperative complications in high-volume centers after lung transplantation, but they have identified these same high-volume centers as best equipped for minimizing adverse effects of complications on short- and long-term survival. Similar associations were identified between postoperative complications and survival in cardiac and renal transplant recipients. ${ }^{9,11}$ These important findings highlight the need for further in-depth analysis into an intriguing aspect of surgical management of complications after high-risk procedures.

Complication types such as infections have been extensively studied in the lung transplantation literature. ${ }^{13,14}$ However, few reports have rigorously examined the overall postoperative complications profile of lung transplant recipients. The purpose of the current study is to identify and create the postoperative complication profile for this population. Further, we sought to investigate the relationship between long-term survival and the way in which it is influenced by each adverse event during the postoperative course. Using the Accordion Severity Grading System (ASGS) to assess severity, we hypothesized that in-hospital, postoperative complications have an independent, negative impact on long-term survival.

\section{METHODS \\ Data Sources}

The University of Pittsburgh Medical Center Transplant Patient Management System database was utilized to identify patients suitable for this study. Prior to extracting data, institutional review board approval was obtained to ensure patient confidentiality. Between January 2007 and October 2013, a comprehensive database on all postoperative complications was prospectively recorded on all 748 patients transplanted at that time. Follow-up included the day of transplantation until death or censoring on August 31, 2015. No patient was lost to follow-up for postoperative complications or mortality.

\section{Data Variables}

All variables were obtained from the University of Pittsburgh Medical Center Cardiothoracic Transplant database. These included, among others, demographics such as comorbidities and diagnosis, characteristics of the surgical procedure, cause of death, and annotated in-hospital postoperative complications occurring within 90 days of surgery. Comorbidities were quantified using the Charlson Comorbidity Index (CCI). ${ }^{15}$ In-hospital complications up to 90 days were included in the analysis to achieve a comprehensive list of complications while excluding medical events that may have been unrelated to the surgery or postoperative course. Postoperative complications that occurred outside of the initial 90 days, including outside-hospital events resulting in readmission, were excluded from this study, owing to the relative ambiguity of their origin. Adverse events were identified via chart review by a dedicated team of database professionals and adjudicated by a lung transplantation surgical-quality committee prior to updating of patient profiles in the database. The date of occurrence was verified as that between the day of surgery and the date of discharge, prior to labeling them as in-hospital postoperative complications.

Following a methodologic approach that paralleled our previous work, 94 postoperative complications were identified based on International Classification of Diseases, 9th edition (ICD-9) diagnostic definitions, as well as findings in the literature, and classified into categories ${ }^{5}$ (Table 1). No additions to the ICD-9 diagnostic codes were necessary. Our primary outcome measure was 1-, 3-, and 5-year overall survival, in keeping with other similar published reports. ${ }^{10,11,16}$

\section{Accordion Severity Grading System}

The extended ASGS version was chosen to classify adverse events. This standardized system quantifies the severity of complications, based on the magnitude of intervention (treatment for complications) necessary to overcome the event. ${ }^{17}$ Details illustrating the course of complications, as well as the extent of treatment the patients underwent, were obtained directly from chart review and included in the database. Grades from the ASGS were weighted based on the burden of total morbidity, as quantified by Porembka and colleagues. ${ }^{18}$ Analysis of the ASGS grades included patients' highest-graded in-hospital postoperative complications, as well as the sum of their weighted ASGS scores, as previously described.

\section{Statistical Analysis}

Cox proportional hazard models were used to test the univariate associations between time-independent covariates, such as demographics, operative characteristics, comorbidities, and post-transplant survival. The Cox model, with time-dependent covariates, mainly postoperative complication categories, was used to test for univariate and multivariate associations with survival. Survival curves were estimated via counting processes in Cox regression models with time-dependent covariates. Data were analyzed using the $\mathrm{R}$ environment for statistical analysis and graphics, ${ }^{19}$ particularly the survival package. ${ }^{20}$ 
TABLE 1. List of complications with 1-, 3-, and 5-year survival

\begin{tabular}{|c|c|c|c|c|c|c|}
\hline \multirow[b]{2}{*}{ Complication type } & \multirow[b]{2}{*}{ No. of type $(\%)$} & \multirow[b]{2}{*}{ n ( $\%$ of 748 patients) } & \multicolumn{3}{|c|}{ Survival $(\%)$} & \multirow[b]{2}{*}{ Hazard ratio $(95 \%$ CI $)$} \\
\hline & & & $1-\mathbf{y}$ & $3-y$ & $5-y$ & \\
\hline None & - & $54(7.22)$ & 94.4 & 86.7 & 73.8 & - \\
\hline Any & $3381(100)$ & $694(92.78)$ & $\begin{array}{c}82.4 \\
P<.01\end{array}$ & $\begin{array}{c}66.9 \\
P=.001\end{array}$ & $\begin{array}{c}53.3 \\
P=.00017\end{array}$ & $2.50(1.40,4.48)$ \\
\hline Renal & $271(8.02)$ & $271(36.23)$ & $\begin{array}{c}66.7 \\
P<.0001\end{array}$ & $\begin{array}{c}49.8 \\
P<.0001\end{array}$ & $\begin{array}{c}35.4 \\
P<.0001\end{array}$ & $2.58(2.05,3.23)$ \\
\hline Hepatic & $48(1.42)$ & $48(6.42)$ & $\begin{array}{c}42.5 \\
P<.0001\end{array}$ & $\begin{array}{c}22.6 \\
P<.0001\end{array}$ & $\begin{array}{c}18.1 \\
P<.0001\end{array}$ & $4.08(2.86,5.82)$ \\
\hline Cardiac & $329(9.73)$ & $268(35.83)$ & $\begin{array}{c}72.4 \\
P<.0001\end{array}$ & $\begin{array}{c}54.4 \\
P<.0001\end{array}$ & $\begin{array}{c}39.5 \\
P<.0001\end{array}$ & $1.95(1.56,2.45)$ \\
\hline Vascular & $114(3.37)$ & $109(14.57)$ & $\begin{array}{c}64.2 \\
P=.001\end{array}$ & $\begin{array}{c}49.1 \\
P=.001\end{array}$ & $\begin{array}{c}29.4 \\
P<.0001\end{array}$ & $2.00(1.52,2.65)$ \\
\hline Neurologic & $115(3.40)$ & $86(11.50)$ & $\begin{array}{c}55.0 \\
P<.0001\end{array}$ & $\begin{array}{c}41.4 \\
P<.0001\end{array}$ & $\begin{array}{c}32.6 \\
P<.0001\end{array}$ & $2.07(1.52,2.81)$ \\
\hline Musculoskeletal & $50(1.48)$ & $48(6.42)$ & $\begin{array}{c}71.7 \\
P<.05\end{array}$ & $\begin{array}{c}42.5 \\
P<.0001\end{array}$ & $\begin{array}{c}27.4 \\
P<.0001\end{array}$ & $2.47(1.70,3.59)$ \\
\hline Pleural space & $517(15.29)$ & $345(46.12)$ & $\begin{array}{c}76.6 \\
P<.0001\end{array}$ & $\begin{array}{c}6.4 \\
P<.0001\end{array}$ & $\begin{array}{c}48.7 \\
P<.0001\end{array}$ & $1.60(1.27,2.00)$ \\
\hline Infectious & $892(26.38)$ & $520(69.52)$ & $\begin{array}{c}82.0 \\
P<.01\end{array}$ & $\begin{array}{c}64.9 \\
P<.0001\end{array}$ & $\begin{array}{c}51.4 \\
P<.001\end{array}$ & $1.65(1.26,2.16)$ \\
\hline Pulmonary & $713(21.09)$ & $536(71.66)$ & $\begin{array}{c}8.8 \\
P<.001\end{array}$ & $\begin{array}{c}65.5 \\
P=.001\end{array}$ & $\begin{array}{c}51.3 \\
P<.001\end{array}$ & $1.57(1.20,2.05)$ \\
\hline Gastrointestinal & $59(1.75)$ & $49(6.55)$ & $\begin{array}{c}6.2 \\
P=.001\end{array}$ & $\begin{array}{c}52.3 \\
P=.01\end{array}$ & $\begin{array}{c}28.7 \\
P=.001\end{array}$ & $1.98(1.35,2.91)$ \\
\hline Psychiatric episode & $134(3.96)$ & $125(16.71)$ & $\begin{array}{c}79.6 \\
P=.057\end{array}$ & $\begin{array}{c}58.7 \\
P<.01\end{array}$ & $\begin{array}{c}44.1 \\
P<.01\end{array}$ & $1.56(1.18,2.05)$ \\
\hline Wound-healing complication & $27(0.80)$ & $27(3.61)$ & $\begin{array}{c}54.3 \\
P<.001\end{array}$ & $\begin{array}{c}4.7 \\
P=.001\end{array}$ & $\begin{array}{c}32.6 \\
P<.01\end{array}$ & $2.22(1.36,3.63)$ \\
\hline Hematologic & $7(0.21)$ & $7(0.94)$ & $\begin{array}{c}24.0 \\
P<.01\end{array}$ & $\begin{array}{c}16.0 \\
P<.01\end{array}$ & $\begin{array}{c}16.0 \\
P<.01\end{array}$ & $4.24(1.75,10.26)$ \\
\hline Endocrine & $36(1.06)$ & $36(4.81)$ & $\begin{array}{c}88.9 \\
P=.608\end{array}$ & $\begin{array}{c}71.6 \\
P=.835\end{array}$ & $\begin{array}{c}6.9 \\
P=.628\end{array}$ & $0.87(0.49,1.55)$ \\
\hline Ear, nose, throat & 69 (2.04) & 67 (8.96) & $\begin{array}{c}85.1 \\
P=.931\end{array}$ & $\begin{array}{c}72.9 \\
P=.661\end{array}$ & $\begin{array}{c}58.1 \\
P=.841\end{array}$ & $0.96(0.64,1.45)$ \\
\hline
\end{tabular}

$\bar{P}$ values are comparing differences in survival between patients with who experienced a complication type versus those who did not (eg, renal vs nonrenal). $C I$, Confidence interval.

\section{RESULTS}

\section{Patient Characteristics}

A total of 748 patients underwent single or double lung transplantation for end-stage lung disease, from January 2007 to October 2013, at our institution. A total of $91 \%$ of our patients were Caucasian. Median follow-up time was 5.4 years (95\% confidence interval [CI] 5.2-5.8 years). Demographics of the cohort and 5-year survival estimates are given in Table 2 . A total of $82.2 \%$ of patients underwent a double lung transplantation, and approximately $21 \%$ of our patients were categorized as obese (body mass index $>30$ ). The mean lung allocation score was 48.2 (standard deviation: 19). Mean intensive care unit stay for our cohort was 10.5 days (standard deviation: 15.3), with a median of 5 days (range $=1-150$ days). Intraoperative cardiopulmonary bypass was utilized in one third of our patients. Fifty nine $(7.9 \%)$ patients required extracorporeal membrane oxygenation. Most indications for transplantation were for idiopathic pulmonary fibrosis $(47.7 \%)$ and chronic obstructive pulmonary disease $(36.2 \%)$.

\section{In-Hospital Postoperative Complication Distribution and Survival Analysis}

The overall postoperative complication distribution of our cohort is summarized in Table 1. Fifty-four (7.22\%) patients had an uneventful postoperative course. A total of 3381 postoperative complications occurred in the remaining $694(92.78 \%)$ patients during the first 90 days postoperatively. The most commonly occurring complication categories were pulmonary $(71.66 \%)$, infectious $(69.52 \%)$, pleural space-related $(46.12 \%)$, renal $(36.23 \%)$, and cardiac $(35.83 \%)$. Figure $1, A$ depicts the difference in survival observed between those without any complications and those who developed at least one complication within 90 days of surgery. Survival at 5 years $(73.8 \%$ vs $53.3 \%$, $P=.00017$; hazard ratio $[\mathrm{HR}] 2.50)$ was greater for 
TABLE 2. Patient demographics

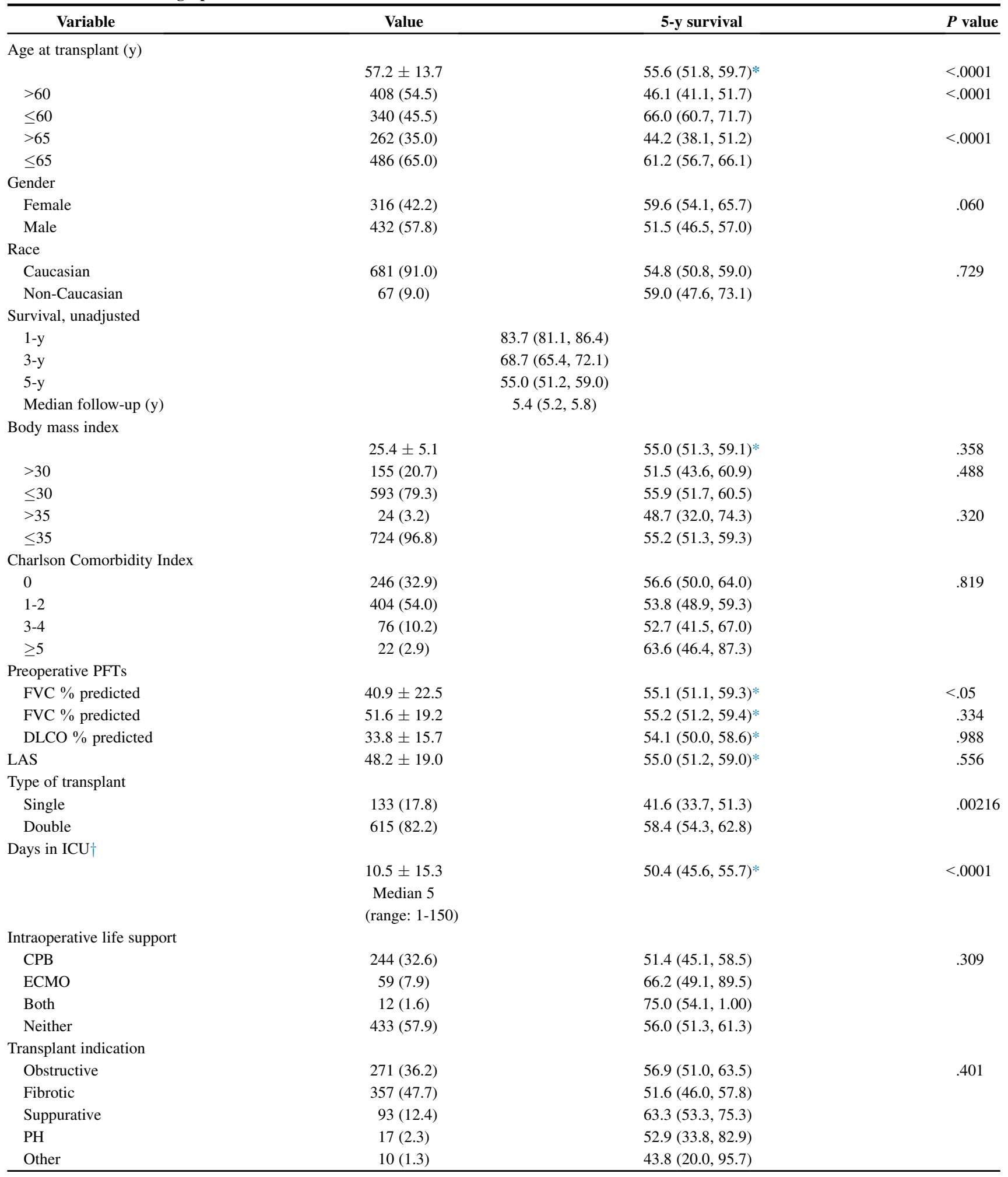

Values are mean \pm standard deviation, $\mathrm{n}(\%)$, or $\%$ (95\% confidence interval), unless otherwise specified. $P$ values are from likelihood ratio tests in Cox models. $P F T$, Pulmonary fibrosis; FVC, forced vital capacity; $D L C O$, diffusion capacity of the lungs for carbon dioxide; $L A S$, Lung Allocation Score; $I C U$, intensive care unit; $C P B$, cardiopulmonary bypass; $E C M O$, extracorporeal membrane oxygenation; $P H$, pulmonary hypertension. *5-year survival estimates for quantitative predictors from univariate Cox models with predictors fixed at mean values. † $†$ Days in ICU are modeled as a time-dependent predictor of mortality. HR $=1.029,95 \%$ CI 1.024, 1.034 . 

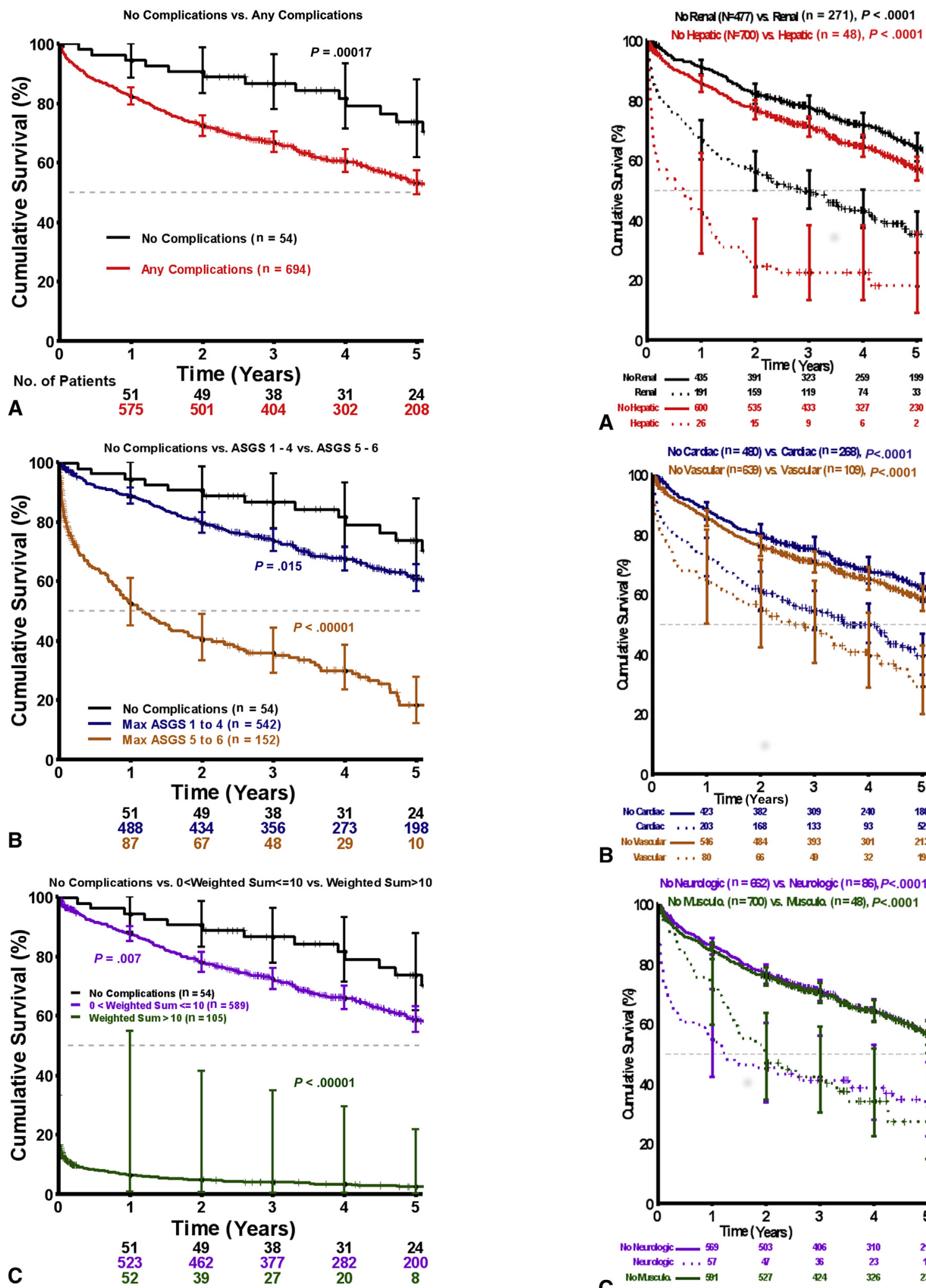

FIGURE 1. A, Survival estimates among patients who have any postoperative complications, versus none; and (B and C) with ASGS grade. ASGS, Accordion Severity Grading System; max, maximum. 
TABLE 3. Distribution of ASGS grades, maximum scores, and weighted sums

\begin{tabular}{lc}
\hline \multicolumn{1}{c}{ Grouping } & $\mathbf{N}(\%)$ \\
\hline ASGS grades & \\
1 & $654(19.34)$ \\
2 & $1392(41.17)$ \\
3 & $586(17.33)$ \\
4 & $470(13.90)$ \\
$5-6$ & $279(8.25)$ \\
Maximum ASGS score & \\
0 (no complication) & $54(7.22)$ \\
1 & $11(1.47)$ \\
2 & $95(12.70)$ \\
3 & $176(23.53)$ \\
4 & $260(34.76)$ \\
$5-6$ & $152(20.32)$ \\
Weighted ASGS score sum & \\
0 & $54(7.22)$ \\
$0<\mathrm{X} \leq 10$ & $589(78.74)$ \\
$>10$ & $105(14.04)$ \\
\hline
\end{tabular}

Values are $\mathrm{n}(\%)$; for grades, $\%$ is of complications; for scores and sum scores, $\%$ is per cohort. ASGS, Accordion Severity Grading System.

individuals who experienced a benign postoperative course; this difference was statistically significant.

Figure 2 depicts the survival curves for the postoperative complications associated with decreases in 5-year survival that have the highest levels of statistical significance. Renal complications were associated with the most statistically significant decrease in 5-year survival, at 35.4\%, compared with $64.4 \%$ in patients without renal complications (HR $2.58,95 \%$ CI 2.05-3.23; $P<.0001)$. Patients who developed hepatic $(18.1 \%$ vs $57.3 \%$, HR $4.08,95 \%$ CI $2.86-5.82$; $P<.0001)$ and cardiac (39.5\% vs $62.3 \%$, HR $1.95,95 \%$ CI $1.56-2.45, P<.0001)$ complications had significantly worse 5-year survival rates after those with renal complications. The 4 complication categories associated with the most statistically significant decreases in 5-year survival after renal, hepatic, and cardiac were: vascular $(29.4 \%$ vs $58.5 \%$, HR 2.00, 95\% CI 1.52-2.65; $P<.0001$ ); neurologic (32.6\% vs $57.1 \%$, HR 2.07, 95\% CI 1.52-2.81); musculoskeletal $(27.4 .0 \%$ vs $56.8 \%$, HR $2.47,95 \%$ CI $1.70-3.59$; $P<.0001)$; and pleural-space events $(48.7 \%$ vs $60.3 \%$, HR 1.60, 95\% CI 1.27-2.00; $P<.0001$ ) (Table 1).

\section{Accordion Severity Grading System Survival Analysis}

The complication ASGS grading distribution is noted in Table 3. The most commonly graded ASGS complication in our cohort was a grade $2(n=1392[41.17 \%])$, followed by grade $3(n=586[17.33 \%])$. Subsequent survival analysis was done with the ASGS system. Patients were first assigned an ASGS score equivalent to their highest ASGS-graded postoperative complication. The mostcommon maximum ASGS grade assigned was $4(n=260$
[34.76\%]), followed by $3(\mathrm{n}=176[23.53 \%])$. Figure 1 , $B$ depicts the survival curves comparing patients who had no complications, versus those with ASGS grades 1 to 4 versus ASGS grades 5 to 6 . A statistically significant difference was found in 5-year survival between patients with no postoperative complications and patients who were assigned ASGS scores 1 to $4(73.8 \%$ vs $61.0 \%$, $P=.015)$. A more drastic statistically significant difference in 5-year survival was noted between patients with no complications and patients whose ASGS score was 5 to 6 (73.8\% vs $18.5 \%, P<.00001)$.

Next, each individual ASGS grade was weighted, based on the system of Porembka and colleagues, ${ }^{18}$ and the sum was calculated. Weighted sums were grouped into categories in Table 3. Most weighted sums were $0<\mathrm{X} \leq 10$ $(78.74 \%)$. Figure 1, $C$ depicts the differences in survival based on weighted ASGS sum. A statistical difference was found in long-term survival between the weighted ASGS sum of 0 (no complications) and weighted sums $0<\mathrm{X} \leq 10(73.8 \%$ vs $58.8 \%, P=.007)$. Compared with patients with no complications, those with an ASGS sum $>10(\mathrm{n}=105)$ exhibited significantly worse 5-year survival $(73.8 \%$ vs $2.5 \%, P<.00001)$. A statistically significant difference in 5-year survival was also noted between those whose weighted ASGS sum was $0<\mathrm{X} \leq 10$ versus $>10(58.8 \%$ vs $2.5 \%, P<.0001)$.

\section{Charlson Comorbidity Index Analysis}

The CCI distribution among our lung transplant-recipient cohort is provided in Table 2. Most of our patients (54\%) had a CCI of 1 to 2, with the next most frequent CCI being $0(32.9 \%)$. The CCI was not found to be a statistically significant predictor of survival $(P=.819)$. Analysis of variance testing confirmed no statistical evidence of any difference in distribution between CCI groups in overall weighted ASGS sum $(P=.969)$ or maximum ASGS $(P=.864)$.

\section{Multivariate Analysis}

The multivariate analysis performed included age at transplantation, year of surgery, type of surgery, and all complication categories. The following predictors were selected in the multivariate analysis of long-term survival: age at transplantation $>65$ years (adjusted HR 1.01; $P=.0012$ ); renal events (adjusted HR 1.70; $P<.0001$ ); cardiac events (adjusted HR 1.29; $P=.037$ ); vascular events (adjusted HR 1.33; $P=.037$ ); and weighted ASGS sum (adjusted HR 1.08; $P=.046$ ). No other complication categories were selected in the multivariate model of long-term survival. The addition of CCI into the multivariate model had no significant effect $(P=.67)$.

\section{DISCUSSION}

This study is the first to systematically evaluate and profile complications that occur after lung transplantation, and 
to relate these data to outcome. According to Van Trigt and colleagues, ${ }^{21}$ in an analysis using the United Network for Organ Sharing database, the 1- and 5-year survival for patients who had undergone lung transplantation decreased from $78 \%$ to $50 \%$. North American 5-year survival in the past decade, per the International Society for Heart and Lung Transplantation, is estimated ${ }^{16}$ at roughly $57 \%$, compared with $45 \%$ in patients who underwent lung transplantation in the period from 1990 to 1997. Although improved, these rates are far lower than those for other organ transplantation, such as the heart, which had a 5 -year survival of $70 \%$ in the same study. As of 2011, the US Renal Disease System annual data report ${ }^{22}$ states a 5 -year survival of $83 \%$. All of these figures indicate the need to identify areas in which improvements may be made in the care of patients undergoing lung transplantation.

\section{Pretransplantation Factors}

Pretransplantation factors, such as comorbidities, have been shown to have an association with survival after cardiothoracic and transplantation procedures. ${ }^{23,24}$ Recently, the presence of diabetes mellitus has been shown to increase death in cystic fibrosis patients who are on the wait list, before lung transplantation, but not to influence survival after lung transplantation. ${ }^{25}$ Although our study was not designed to investigate pretransplantation status and the incidence of postoperative events, we were able to calculate the CCI for each patient, to quantify comorbidity profiles.

Based on our analysis, CCI was not found to be a predictor of long-term survival. Moreover, no statistical differences were found in distribution of weighted ASGS sums and maximum ASGS scores within the various CCI groups. This homogeneity in the ASGS distribution per CCI group indicates that CCI may not be suitable for predicting the postoperative course of lung transplant recipients. The lack of an association between CCI and survival after lung transplantation was further confirmed in our study with our multivariate model.

\section{Incidence of In-Hospital Postoperative Complications}

The incidence of in-hospital postoperative complications in patients who have undergone lung transplantation in our study was $>90 \%$. This figure may be staggering, compared with other literature accounts of surgical complications. However, many of these studies use a voluntary reporting system. Moreover, many investigators focus mainly on the major adverse events categories, such as infectious profiles, especially as they pertain to the immunocompromised state of the recipient, acute and chronic rejection, airway complications, renal complications, diabetes, and diaphragmatic paralysis. In 2002, Chhajed and colleagues ${ }^{14}$ published a review that explored several other categories not often studied. Our main goal was to provide a comprehensive profile for any possible complications. Therefore, we selected patients from a time period when postoperative complications had been meticulously recorded, adjudicated, and documented in our database.

Among the patients who developed complications in this study, 5-year survival remained consistent with published survival rates. ${ }^{17}$ However, compared with patients who had an uneventful postoperative course, the difference in 5 -year survival rate was significantly longer. Our results indicate that 90-day postoperative complications have a significant association with long-term survival and can serve as a determinant for patient outcomes.

These results are further highlighted by the impact of having specific types of postoperative complications. In our cohort, the 7 complication categories that had the most significant adverse impact on long-term survival were renal, hepatic, cardiac, vascular, neurologic, musculoskeletal, and pleural space. These complications make up $>47 \%$ of all our complications. Several other categories, including infectious, pulmonary, gastrointestinal, and psychiatric, also showed statistical significance and need to be further studied. This finding is an important step toward defining associations between donor and recipient characteristics that may significantly affect outcomes.

\section{Survival Analysis With the ASGS}

The ASGS was used in our analysis to assess the severity of each complication, based on the extent of the intervention necessary to overcome an adverse event. During data analysis, we observed the importance of using this systematic approach to further quantify and categorize each complication, owing to the potential complex course each may undergo. We recognized that although every complication type may occur within the context of the standard of care, each individual event may require a different level of intervention.

Moreover, each institution, as well as every medical care provider, has preferences for treatment choice. This variability in postoperative complication identification and treatment algorithm across institutions was previously identified to be a major contributing factor to failure-to-rescue rates, which were found to be significantly higher in institutions with higher mortality after major surgery. ${ }^{6,7}$ In the setting of a single institution, we hope to strengthen the generalizability of our results with the analysis of each complication with a well documented standardized system to minimize the effects of variability in other centers. Implementing the ASGS system may give physicians in other institutions the ability to apply our results to their practice.

Our analysis of the ASGS system was done using the maximum score, as well as the weighted sum of all postoperative events. In our cohort, a disparity was seen in the patients with a maximum ASGS score of $\geq 5$ and a weighted 
ASGS sum $>10$. These thresholds represent essentially different populations. This uneven distribution can be attributed to the numerous combinations possible that lead to the respective ASGS status.

Using the maximum score allows us to identify the specific goal of decreasing all complications that result in "multisystem organ failure or a complication that required the following: an invasive procedure under general anesthesia or requiring intensive care unit admittance," as that is the singular way to meet that threshold. ${ }^{17}$ However, the weighted ASGS sum threshold of $>10$ can be met through an infinite combination of many lower-scoring events, and a handful of higher-scoring incidences. ${ }^{18}$ This important distinction between the 2 ASGS methods may be the reason that only the weighted ASGS sum strategy was selected by our multivariate analysis on long-term survival. Because all complications contributed to the weighted sum, this method may provide more insight into the entire postoperative course. Ultimately, a prospective study investigating both methods is needed to compare the benefits of both systems.

\section{Multivariate Model}

Some of our multivariate analysis findings support results published in the literature. ${ }^{22}$ Mainly, better survival is associated with double lung transplantation. However, decreased survival was associated with being aged $>65$ years. This finding differs from results of several studies that did not find increased age to have a significant impact on short- or long-term outcomes. ${ }^{26,27}$

Moreover, renal, cardiac, and vascular complications were found to be significant predictors of long-term survival, along with the weighted ASGS sum. Analysis of these complication types reveals a higher frequency of higher ASGS grades. All renal complications were labeled as "acute renal dysfunction" events, and 33\% (88 of 271) resulted in an ASGS score of $\geq 4$, representing at least the need for dialysis as treatment. A total of 68 of $271(25 \%)$ of all renal events were graded 5 or 6 , signifying multiorgan system failure. When these 3 complication categories were isolated, 185 of $748(25 \%)$ of our patients experienced $\geq 1$ event in $\geq 2$ of these complication categories. Because of concurrent organ failure, attributing the association with decreased 5-year survival after lung transplantation to any specific one of these complication categories is difficult. Further studies are needed to definitively study this association.

The ASGS score, either as a weighted sum or a maximum score, can be used to predict long-term survival of post-lung transplantation patients. Although these scores can be assigned only after a complication has been resolved, thereby limiting the real-time applicability of the ASGS system, it can be effectively used as a retrospective tool, to identify possible complications that may affect survival, as seen with renal complications in our cohort.
Moreover, specific patients that meet these thresholds may need to be subjected to more-meticulous monitoring after transplantation.

These results highlight the need to implement procedurespecific postoperative monitoring and intervention protocols, with the ultimate goal of eliminating these types of postoperative adverse events. Clinical pathways have been found to be effective in several other medical fields. ${ }^{28-31}$ Future studies are needed to measure the success of these pathways in lung transplantation surgery, but this study shows promise in this area.

Our study has several important attributes. With 748 eligible patients, this series offers one of the largest evaluations of patients undergoing lung transplantation that has a specific focus on their postoperative course. Moreover, the reporting of any postoperative complication was not restricted to self-reporting by the medical team. Complications were maintained by a team of database professionals and were additionally adjudicated by a panel of cardiothoracic transplant surgeons before finalizing their addition to the database.

\section{Limitations}

We acknowledge several limitations in this study. First, it was conducted retrospectively and within a single institution. The retrospective nature invariably subjects the current study to selection, as well as information, bias. These biases were actively addressed through implementation of various data checkpoints, with multiple investigators. Nonetheless, such bias limits the generalizability of our findings.

\section{CONCLUSIONS}

With appropriate patient selection and contemporary surgical techniques, vigilant postoperative management and avoidance of adverse events may potentially offer patients better long-term outcomes. This study demonstrates the independent negative association between in-hospital, postoperative complications and long-term survival in patients who have undergone lung transplantation. The overall 90-day postoperative course has an influence on long-term survival.

Specifically, both the severity of the intervention needed to overcome a complication and the occurrence of many less-severe complications have negative impacts regarding long-term survival. These results, however, support the need to establish best-practice guidelines outlining the best way to avoid complications in these patients, particularly during this initial crucial period. The next step is to identify interventions that effectively reduce the incidence, as well as severity, of in-hospital, postoperative complications, to potentially improve long-term survival in these recipients.

\section{Conflict of Interest Statement}

Authors have nothing to disclose with regard to commercial support. 


\section{References}

1. Yusen RD. Survival and quality of life of patients undergoing lung transplant. Clin Chest Med. 2011;32:253-64.

2. Yusen RD, Shearon TH, Qian Y, Kotloff R, Barr ML, Sweet S, et al. Lung transplantation in the United States, 1999-2008. Am J Transplant. 2010;10: 1047-68.

3. Valapour M, Skeans MA, Heubner BM, Smith JM, Hertz MI, Edwards LB, et al. OPTN/SRTR 2013 Annual Data Report: Lung. Am J Transplant. 2015;15:1-28.

4. Bach PB, Cramer LD, Schrag D, Downey RJ, Gelfand SE, Begg CB. The influence of hospital volume on survival after resection for lung cancer. $N$ Engl J Med. 2001;345:181-8.

5. Rueth NM, Parsons HM, Habermann EB, Groth SS, Virnig BA, Tuttle TM, et al. The long-term impact of surgical complications after resection of stage I nonsmall cell lung cancer: a population-based survival analysis. Ann Surg. 2011;254:368-74.

6. Ghaferi AA, Birkmeyer JD, Dimick JB. Complications, failure to rescue, and mortality with major inpatient surgery in Medicare patients. Ann Surg. 2009; 250:1029-34

7. Ghaferi AA, Birkmeyer JD, Dimick JB. Variation in hospital mortality associated with inpatient surgery. N Engl J Med. 2009;361:1368-75.

8. Livingston EH, Cao J. Procedure volume as a predictor of surgical outcomes. JAMA. 2010;304:95-7.

9. Rajagopal K, Lima B, Petersen RP, Mesis RG, Daneshmand MA, Lemaire A, et al. Infectious complications in extended criteria heart transplantation. J Heart Lung Transplant. 2008;27:1217-21.

10. Kilic A, George TJ, Beaty CA, Merlo CA, Conte JV, Shah AS. The effect of center volume on the incidence of postoperative complications and their impact on survival after lung transplantation. J Thorac Cardiovasc Surg. 2012;144: 1502-8; discussion 1508-9.

11. Campos Hernandez JP, Gomez Gomez E, Carrasco Valiente J, Marques Lopez FJ, Ruiz Garcia J, Angelada Curado FJ, et al. Influence of surgical complications on kidney graft survival in recipients of simultaneous pancreas kidney transplantation. Transplant Proc. 2015;47:112-6.

12. Thabut G, Christie JD, Kremers WK, Fournier M, Halpern SD. Survival differences following lung transplantation among US transplant centers. JAMA. 2010;304:53-60.

13. Schuurmans MM, Benden C, Inci I. Practical approach to early postoperative management of lung transplant recipients. Swiss Med Wkly. 2013;143: W 13773 .

14. Chhajed PN, Tamm M, Malouf MA, Glanvaille AR. Lung transplantation: management and complications. Indian J Chest Dis Allied Sci. 2002;44:31-43.

15. Austin SR, Wong YN, Uzzo RG, Beck JR, Egleston BL. Why summary comorbidity measures such as the Charlson Comorbidity Index and Elixhauser Score work. Med Care. 2015;53:e65-72.

16. International Society for Heart and Lung Transplantation. ISHLT Transplant Registry Quarterly Reports for Lung in North America. Available at: https://www. ishlt.org/registries/quarterlyDataReportResults.asp?organ =LU\&rptType =all\& continent $=4$

17. Strasberg SM, Linehan DC, Hawkins WG. The accordion severity grading system of surgical complications. Ann Surg. 2009;250:177-86.

18. Porembka MR, Hall Bl, Hirbe M, Strasberg SM. Quantitative weighting of postoperative complications based on the accordion severity grading system: demonstration of potential impact using the American College of Surgeons National Surgical Quality Improvement Program. J Am Coll Surg. 2010;210: 286-98.

19. Ihaka R, Gentleman R. A language for data analysis and graphics. J Comput Graph Stat. 1996;5:299-314.

20. Therneau TM, Grambsch PM. Modeling survival data: extending the Cox model. (Statistics for Biology and Health). New York, NY: Springer; 2000.

21. Van Trigt P, Davis RD, Shaeffer GS, Gaynor JW, Landolfo KP, Higginbotham MB. Survival benefits of heart and lung transplantation. Ann Surg. 1996;223:576-84.

22. United States Renal Data System. United States Renal Data System Annual Report: Transplant. Available at: http://www.usrds.org/adr.aspx.

23. Battafarano RJ, Piccirillo JF, Meyers BF, Hsu HS, Guthrie TJ, Cooper JD, et al. Impact of comorbidity on survival after surgical resection in patients with stage I non-small cell lung cancer. J Thorac Cardiovasc Surg. 2002;123:280-7.

24. Hernandez JC, Lok AS, Marrero JA. Modified Charlson Comorbidity Index for predicting survival after liver transplantation. Liver Transplant. 2007;13: 1515-20.
25. Hayes D, Patel AV, Black SM, McCoy KS, Kirkby S, Tobias JD, et al. Influence of diabetes on survival in patients with cystic fibrosis before and after lung transplantation. J Thorac Cardiovasc Surg. 2015;150:707-13.e2.

26. Schaffer JM, Singh SK, Reitz BA, Zamanian RT, Mallidi HR. Single- vs doublelung transplantation in patients with chronic obstructive pulmonary disease and idiopathic pulmonary fibrosis since the implementation of lung allocation based on medical need. JAMA. 2015;313:936-48.

27. Genao L, Whitson HE, Zaas D, Sanders LL, Schmader KE. Functional status after lung transplantation in older adults in the post-allocation score era. Am J Transplant. 2013;13:157-66.

28. Tomaszek SC, Fibla JJ, Dierkhising RA, Scott JP, Schen KR, Wigle DA, et al Outcome of lung transplantation in elderly recipients. Eur $J$ Cardiothorac Surg. 2011;39:726-31.

29. Vigneswaran WT, Bhorade S, Wolfe M, Pelletiere K, Garrity ER. Clinical pathway after lung transplantation shortens hospital length of stay without affecting outcome. Int Surg. 2007;92:93-8.

30. Pitt HA, Murray KP, Bowman HM, Coleman J, Gordon TA, Yeo CJ, et al. Clinical pathway implementation improves outcomes for complex biliary surgery Surgery. 1999;126:751-6; discussion 756-8.

31. Collier PE. Do clinical pathways for major vascular surgery improve outcomes and reduce cost? J Vasc Surg. 1997;26:179-85.

Key Words: lung transplantation, accordion severity grading system, outcomes, postoperative care, survival, surgical complications

\section{Discussion}

Dr Michael Smith (Phoenix, Ariz). Congratulations on your work; you did a wonderful job of presenting the data and representing your coauthors well. I would also like to thank you for getting me the manuscript well in advance for my preparation for this discussion. I found the manuscript and the presentation to be quite intriguing.

It was a well organized effort to try to quantify and categorize the number of complications and their impact on longterm survival after lung transplantation. I found the $>90 \%$ complication rate after lung transplantation to be quite astonishing, but I realized that when you look at these complications closely, the data are probably accurate for most of our experiences with these challenging patients. Your study does a great job of pointing out the impact of postoperative complications on long-term survival in this group of patients.

I do have a few questions, mainly regarding the methods and the design of the study. First, I think the use of the ICD-9 code can be limiting, in terms of looking at outcomes after lung transplantation, given that there are no specific codes that I am aware of for important postoperative complications after lung transplantation, such as primary graft dysfunction (PGD) and acute rejection. In many studies, severe PGD is one of the factors contributing most strongly to poor perioperative survival, and in turn, long-term survival for those that survive this postoperative insult. In your series, it is difficult to see where this complication fits. So, my first question is: Under which category was this complication of severe (grade-3) PGD placed, and do you have a sense for what the 1- and 5-year survival was for this group of patients? 
Dr Ernest Chan. I believe that in our list of complications, we actually did not include this in our analysis. Having said that, we did use the ICD codes to define certain complications, but there are some that I can explain now that did not help us much. They just gave us guidelines. One example is respiratory insufficiency versus respiratory failure. So, drawing from the literature as well as the ICD codes, we came up with a string of guidelines for that complication. For instance, we deemed the complication to be respiratory failure if patients were on the ventilator for $>72$ hours, or if they needed to be reintubated. So, I believe that for patients who did have PGD, some would be captured by using respiratory failure as a complication.

Dr Smith. Certainly. It may be worthwhile to discuss that further. Some of the early, recurrent, acute rejection episodes are associated with development of early chronic rejection, and therefore poor long-term survival after lung transplantation. Yet, I do not see evidence of that complication in your series, which I suspect may be related also to a lack of a specific ICD-9 code for that.

I thought the systematic categorization of complications into the ASGS was reasonable, but may also have some limitations with regard to the importance of distinguishing the triggering complicating event. It is difficult to get a sense for the timing or sequence of these complications. Understandably, this is more difficult to do, but to me, your title seems to suggest that, with the ripple effect, one complication usually begets another complication, which begets another, which begets another, and so on. With that said, it is not clear to me that the ASGS captures this well, and in many instances, poor allograft function or severe PGD is the first domino to fall that leads to other organ system dysfunction and complications, such as renal, cardiovascular, and musculoskeletal complications, especially if we are talking about postoperative myopathy and deconditioning.

So, what the ASGS seems to prioritize is the severity of a complication based on the level of invasiveness of the intervention required to treat it. With that in mind, I wonder how the ASGS would organize and prioritize the complications of an unfortunate, but classic, postoperative lung transplant patient who developed severe PGD of grade 3, which does not require extracorporeal membrane oxygenation salvage, but needs prolonged ventilator support for several days. And what if that patient has early cardiovascular dysfunction requiring inotrope and pressor support long enough to develop acute renal failure requiring dialysis, and of course, intensive care unit myopathy and deconditioning? I suspect that the ASGS would recognize and categorize all these complications, but what would it give the priority to, based upon the fact that the PGD is a triggering event, but only required, for example, prolonged vent weaning and diuresis, while acute renal failure was what required the most-invasive support via intervention with hemodialysis?

I would like to get your thoughts on that question, and then just one other question is: Was there any correlation between lung allocation score and the complication rates and long-term survival. Once again, congratulations on your work. I would also like to thank the association for asking me to discuss the paper.

Dr Ernest Chan. For your second question-for the lung allocation score, we actually did not look at the correlation between the complication rate and this score, so that is something we should definitely explore. In terms of your other question, the reason that we used the ASGS score in 2 ways for our patients was that we were not sure whether using the maximum ASGS score or the weighted sum would be most appropriate. So, in your example of a patient who had PGD and then had renal failure, we believe that using the weighted sum would encompass the entire postoperative course, as opposed to just their maximum ASGS score.

Dr Smith. In your model, did you account for year as a surrogate for the temporal changes that you noted in the Limitations section?

Dr Chan. We included date of surgery in our model.

Dr Smith. That is a very simple way just to try to control for some of the variations you will have in time. 